\title{
Realizando lo Imaginado. La formación de una política urbana desde la investigación universitaria
}

\author{
Ferrer, Mercedes* \\ Quintero, Carolina**
}

\section{Resumen}

El objeto de este trabajo es presentar la metodología utilizada y los resultados de una investigación aplicada, desarrollada desde la Universidad del Zulia (LUZ), cuyo objetivo fue promover la formación de una política urbana bottom up y una nueva cultura urbana asumiendo en forma colectiva la rehabilitación de un espacio emblemático de la ciudad, el Malecón de Maracaibo. Para construir este pacto por la ciudad o política urbana, entendida como el resultado de soluciones democráticas a problemas sentidos por la comunidad, el grupo de investigación implantó un proceso dialogante y deliberativo con la participación de diferentes actores sociales interesados e involucrados en la recuperación del área central de Maracaibo. El proceso se estructuró en tres fases, Fase 1: Planificación de la Gestión (formulación consensuada de la política y de la estrategia operativa), Fase 2: Construcción de Consensos (o visión compartida) y Fase 3: Ejecución del Convenio de Gestión. Esta última Fase se desarrolló en dos etapas, Etapa 1: Compromiso de participación (firma del Convenio de Gestión) y Etapa 2: Corresponsabilidad en la Gestión (Ejecución del Convenio). Del proceso desarrollado destacan cuatro conclusiones: el modelo de gestión diseñado e implantado concilió la gestión publica con la iniciativa privada y, generó un aprendizaje desde la experiencia donde, actores tradicionalmente en conflicto y compitiendo para impulsar proyectos individuales se comprometieron, a través de la firma de un Convenio de Gestión Estratégica, a rehabilitar el Malecón de Maracaibo colectivamente; el potencial estratégico de la investigación aplicada para generar impacto social; la emergencia de una nueva praxis universitaria posfuncionalista que integra investigación, docencia y extensión como clave del quehacer académico y, la necesidad de involucrar a diferentes actores sociales en el proceso de formación de la política urbana para desarrollar comunidades y ciudades sostenibles.

Palabras clave: Política urbana, patrimonio, rehabilitación, modelo de gestión.

Recibido: 02-01-22 . Aceptado: 02-08-01

* $\quad$ Arquitecto, Master en Filosofía: en Planificación Urbana - Regional, candidata a doctor en Ciencia Política. Profesora e Investigadora Titular de LUZ. Autora de varios artículos publicados en revistas arbitradas. Ponente en diversos eventos nacionales e internacionales.

E. mail: mferrer_98yahoo.com

** $\quad$ Arquitecto, Especialista en Gestión Urbana. Profesora e Investigadora Asistente de LUZ.

E. mail: carolaquinsa@telcel.net.ve 


\section{Doing the Unimaginable: The Formation of Urban Policy from University Research}

\section{Abstract}

The purpose of this paper is to present the methodology utilized and the results of applied research developed in the University of Zulia (LUZ), the objective of which was to promote the formation of bottom-up urban policy and a new urban culture related to the collective rehabilitation of a historic urban space, el Malecon in Maracaibo. In order to develop this united policy in the city , understood as the result of democratic solutions to community problems, the research group proposed a process of dialogue and deliberation with the participation of all the different social actors interested and involved in the recovery of this central Maracaibo area. The process was structured in three phases: Phase 1; Management Planning (consensus formulation as to policy and operative strategy), Phase 2; Construction of consensus (shared vision), and Phase 3; Execution of the management consensus. This last phase was developed in two stages: Stage 1: the promise to participate (participation covenant) and Stage 2: co-responsibility in management (execution of the covenant). Four conclusions resulted from this development: the management model that was designed and implemented reconciled public management with private initiative and generated a learning experience where actors who are traditionally in conflict and competing in the promotion of individual projects came together through the signing of a Strategic Management Agreement and rehabilitated EI Malecon collectively; the strategic potential of the applied research to generate social impact; the emergence of a new post-functionalist university praxis that integrates research, teaching and extension as key academic functions; and the need to involve different social actors in the process of urban policy formation for the development of sustainable communities and cities.

Key words: Urban policy, patrimony, rehabilitation, management model.

\section{Introducción}

La necesidad de rehabilitar y proteger el patrimonio cultural representado por edificaciones, espacios públicos y obras de arte, surge hoy como actividad fundamental en medio de los procesos de transformación de la ciudad y, del caos desatado por el terrorismo que, según Cruz (2001), amenaza por destruir el legado cultural de la humanidad y los símbolos de identidad de los pueblos. Las demandas de conservación del hecho patrimonial son consecuencia de la necesidad de reforzar la identidad de la población, por ser exponente de la cultura del lugar $y$, de calidad de vida, además de generar una importante articulación entre residentes y visitantes. En este contexto, dos factores son clave para la conservación del patrimonio, la participación plural de diferentes agentes sociales y el sentido de afirmación colectiva, los cuales permiten considerar el patrimonio como recurso fundamental para la cohesión social, tema que debe ser atendido, tanto en el presente como en el futuro, por la universidad posfuncionalista (que asume la investigación, la docencia y la extensión como actividades inseparables) y plural, ya que el terrorismo y el fundamentalismo están generando un nuevo entorno que se mueve entre "civilización y barbarie" (Cruz, 2001). 
La reflexión sobre la identidad cultural de los pueblos y colectivos sociales $y$, la defensa de los valores patrimoniales que los representan, se encuentra en el corazón mismo del debate actual ya que, en este momento de transformaciones profundas, según Castells (1998), la oposición entre globalización e identidad está dando forma a nuestro mundo y a nuestras vidas. Esta situación se refleja también según Cruz (2001), en la arquitectura de hoy, que se debate entre la presencia de lenguajes internacionales, globalizadores y la búsqueda de idiomas locales. Según Fernández (1995:5), la crisis de los valores culturales, de la memoria y del patrimonio arquitectónico, se agudiza frente a los procesos acelerados de construcción y producción del hábitat en el contexto metropolitano contemporáneo. En este sentido, Precedo (1996) señala que, en la nueva red de flujos intraurbanos donde predominan procesos centrífugos sobre los centrípetos, muchas áreas centrales están siendo afectadas por procesos de obsolescencia urbana que es más acusado en los centros históricos de las ciudades. Para contrarrestar estas tendencias, el proyecto posmoderno según Fernández (1995), asume una postura conservadora en defensa del patrimonio cultural y natural y, apuesta por una intervención critica e interpretativa de los conjuntos históricos, para que puedan recuperar su plenitud funcional y social.

En este contexto, el objeto de este trabajo es presentar la metodología utilizada y los resultados de una investigación aplicada, desarrollada desde la Universidad del Zulia (LUZ), cuyo objetivo fue promover la formación de una política urbana bottom upy una nueva cultura urbana asumiendo en forma colectiva la rehabilitación de un espacio emblemático de la ciudad, el Malecón, conjunto urbano del área central de la ciudad de Maracaibo (capital del Estado Zulia, ubicada en el occidente de Venezuela), que tiene un alto valor patrimonial y simbólico. Para construir este pacto por la ciudad, el grupo de investigación implantó un proceso dialogante y deliberativo con la participación de diferentes actores sociales interesados e involucrados en la recuperación del área central de Maracaibo. Se seleccionó este espacio y conjunto urbano del área central de Maracaibo por ser un testimonio fundamental de la historia de la ciudad, ya que forma parte de su núcleo fundacional desde donde todavía se vivencia el Lago, escenario natural que dio origen a Maracaibo.

Este conjunto urbano (edificaciones y espacio público emblemáticos), se ubica cronológicamente entre dos siglos, finales del XIX e inicios del XX, periodo en el cual Maracaibo era una ciudad portuaria de vanguardia, con una economía agroexportadora floreciente $y$, aunque actualmente esta muy deteriorado conserva un alto valor simbólico y patrimonial como imagen de la ciudad. Por estas características se seleccionó concertadamente el Malecón como la fase inicial de la recuperación del borde costero de Maracaibo y, para potenciar su imagen como símbolo de identidad de la ciudad, dinamizar la actividad turística y comercial del área central y atraer inversiones, lo cual tendrá impacto en la reactivación y generación de empleo local. Por otro lado, el modelo de gestión implantado, que per- 
mitió asumir la recuperación de El Malecón como estrategia colectiva, viabilizó la formación de una política urbana bottom up desde la investigación universitaria. Esta investigación constituye una experiencia innovadora y piloto en la praxis universitaria de LUZ, generadora de impacto social y con alto potencial de replicabilidad.

\section{Antecedentes y breve reseña del proceso}

El proyecto de investigación se desarrolló en dos etapas, por un grupo de docentes e investigadores de la Facultad de Arquitectura y Diseño de la Universidad del Zulia, FADLUZ. Este grupo de investigación que actualmente desarrolla la segunda etapa de la investigación, trabajó previamente en actividades de extensión ${ }^{1}$. La experiencia obtenida y la praxis desarrollada fue clave en la formulación de la investigación, en diciembre de 1998. La primera etapa se denominó, "MARACAIBO ENTRE DOS SIGLOS UNA CIUDAD DE VANGUARDIA" $y$, la segunda, todavía en curso, "POLÍTICAS URBANAS EN EL GOBIERNO LOCAL". El objetivo fundamental de la primera etapa fue promover una nueva cultura urbana desde la universidad, involucran- do en ella a diferentes agentes urbanos. Esta primera etapa de la investigación se inició en Julio de 1999 y concluyó en Julio de 2000, con la entrega del proyecto del Convenio de Gestión Estratégica para Rehabilitar el Malecón y las bases para llamar a un Concurso Internacional de Ideas. Estos dos productos fueron el resultado del proceso dialogante o de deliberación democrática (Crozier, 1996; Giddens, 1996; Uvalle, 2001) y, de concertación, realizado con doce instituciones, privadas y públicas, pertenecientes estas últimas a los tres niveles de gobierno del territorio: local, regional y nacional. En Diciembre de 2000, se formuló la segunda etapa del proyecto de investigación ya mencionado anteriormente, Políticas Urbanas en el Gobierno Local ${ }^{2}$.

Es importante señalar como hito importante de este proceso que, el consenso para rehabilitar colectivamente EI Malecón que se denominó pacto por la ciudad, fue el acuerdo más importante logrado en el Seminario: "La Rehabilitación de El Malecón de Maracaibo como estrategia colectiva", realizado con ese objetivo en Marzo de 2000 en Maracaibo. Este Seminario fue promovido y financiado por el Centro Rafael Urdaneta S.A. (CRUSA), la Cámara de Comercio de Maracaibo y el grupo de investigación de la FADLUZ. En

1 En la Dirección de Extensión de la Facultad de Arquitectura y Diseño de la Universidad del Zulia (FADLUZ).

2 El financiamiento se obtuvo, al igual que en la primera etapa, a través de el Consejo de Desarrollo Científico Humanístico de LUZ, (CONDES). Este proyecto, que se inició en Mayo de 2001, forma parte del Programa de Investigación (interfacultades y multidisciplinario): Políticas y Administración Subnacional, adscrito al Centro de Estudios de la Empresa de la Facultad de Ciencias Económicas y Sociales de LUZ. 
el Seminario participaron, presentando sus planes y proyectos para El Malecón, las instituciones que hoy suscriben el Convenio.

El 21.12.2000 se firmó el CONVENIO ESTRATÉGICO PARA LA REHABLLITACION DE EL MALECÓN DE MARACAIBO Y SU ENTORNO INMEDIATO, que incluyó tres objetivos sobre los que han trabajado concertadamente las doce instituciones: 1. Definir las directrices de desarrollo para El Malecón, 2. Llamar a un Concurso Internacional de Ideas para Rehabilitar el Malecón y 3. Declarar El Malecón de Maracaibo patrimonio regional. En marzo de 2001 se inició la divulgación y el llamado al Concurso Internacional de Ideas, en el cual participaron equipos de arquitectos de varios países: América Latina, Estados Unidos y Europa. El 21 de Junio de ese año se recibieron catorce propuestas que fueron evaluadas por un jurado seleccionado por las instituciones integrantes del convenio e integrado por expertos arquitectos, urbanistas y paisajistas, nacionales y extranjeros. El 6 de Julio de 2001 el jurado presentó el veredicto del concurso en acto público, donde se expusieron las catorce. El proyecto ganador, elaborado por una empresa de arquitectos de Caracas, esta actualmente en proceso de contratación por el Centro Rafael Urdaneta S.A., CRUSA.

La divulgación del CONCURSO INTERNACIONAL DE IDEAS, se realizó a través de la web para lo cual se crearon dos páginas que fueron diseñadas por profesores de la FAD y de Ingeniería de LUZ: www.geocities.com/malecondemaracaibo/ y www.luz.ve/malecón. En ellas se ubicó la información necesaria para que los concursantes pudieran participar proponiendo sus ideas. A través de estas páginas se mantendrá informada a la comunidad nacional e internacional del avance de esta experiencia y, como parte de ello se podrá consultar el veredicto del concurso, los proyectos ganadores y el avance del proyecto contratado.

Paralelamente a este esfuerzo se ha desarrollado un proceso comunicacional importante con tres objetivos básicos: generar una matriz de opinión favorable -capital social- (Fukuyama, 1996), concientizar a la opinión pública para que asuma y defienda el proyecto $y$, desarrollar un proceso de formación potenciando los recursos humanos -capital intelectual(Gates, 1999) disponibles. En este sentido, en Junio de 2001, el Arq. Nicolas Drouin de la Comunidad de Burdeos, Francia, asesor del proyecto y del proceso de gestión del concurso, dictó una conferencia en la FAD, sobre la recuperación de las riberas de río en Burdeos y, el 4 de Julio de 2001, día del Arquitecto, se realizó en el auditorio de la FADLUZ la "Conferencia Internacional sobre Gestión Urbana", cuyos conferencistas fueron los jurados del concurso internacional.

\section{Encuadre conceptual: un pasado con futuro}

Este trabajo se apoya en cuatro conceptos clave: investigación aplicada, patrimonio-cohesión social, rehabilitación de centros históricos y, política públicas y urbanas. 


\subsection{Investigación aplicada, estrategia de la universidad en el siglo XXI}

La globalización, la internacionalización de las profesiones, la cooperación entre países culturalmente afines (Huntington, citado por Ferrer; 1998), la asunción del conocimiento como impulsor de la economía y creador de riqueza (Tofler citado por Ferrer; 1998), que le confiere protagonismo a las universidades y el desarrollo de sistemas informacionales globales, son aspectos clave para identificar las tendencias en la investigación: la creciente demanda de investigación aplicada o problem solving (agenda) y, el desarrollo de investigación en red e internacionalizada (virtuales), para sustentar estudios de IV y V nivel. En este sentido, la política de fortalecimiento de la investigación aplicada o extensiva se apoya en tres aspectos importantes referidos el primero, a la necesidad de mejorar la actuación de la universidad -relación con el medio- y sus indicadores para competir con éxito y lograr mejor presupuesto y ranqueo; el segundo, la necesidad de obtener financiamiento alternativo a través del acceso a fuentes distintas a las internas, dadas las dificultades financieras por las que atraviesan estas instituciones recurrentemente y, el tercero, a la necesidad de cambiar el modelo de gestión académica y administrativa de la universidad ante la implantación de la carrera académica y la acreditación de las universidades en Venezuela.

Por otro lado, la investigación extensiva es el medio por excelencia de la universidad posfuncionalista, plural y postburocratica (que viabiliza formas de organización horizontal asegurando per- tinencia social), con rentabilidad social (Ferrer, 1999), para vincularse con el medio, resolver problemas de la sociedad a corto plazo, contextualizar la investigación (responsabilidad social y pertinencia) y promover la actualización y formación docente, a partir de la vinculación teoría-praxis. La visión plural de la investigación permite la coexistencia de distintas formas de investigación (Ferrer, 1998) y viabiliza el surgimiento de un nuevo proyecto ético-político universitario (Licha, 1992). La investigación extensiva integra lo social con lo económico y lo político. Es una investigación estratégica porque, en el marco de un sistema de información e inteligencia continua, produce ajustes que cambian la realidad y estos a su vez producen nuevos inputs para investigar $y$, tiene un alto potencial para generar impacto social, porque resuelve problemas, promueve el desarrollo institucional y profundiza la vinculación con el entorno.

\subsection{Patrimonio y Cohesión Social}

Para Rivière (en Fernández-Baca, 1999), el patrimonio histórico lo constituyen aquellos bienes materiales e inmateriales sobre los que, como en un espejo, la población se contempla para reconocerse, donde busca explicación a su territorio, donde esta enraizada y en el que se sucederán los pueblos que le precedieron. Un espejo que la gente ofrece a sus huéspedes para hacerse entender, con respeto a su trabajo, a sus formas de comportamiento y a su intimidad. De esta definición destaca según Fernández-Baca (1999), la existencia de un sentido de identidad o diferenciación que quiere 
transmitirse a otros. Es por esto que en la actualidad el patrimonio ocupa un lugar importante y demandado por los colectivos sociales, en respuesta a la necesidad de reforzar su identidad ante el proceso de macdonalización (uniformalización). Berlinches (1995: 73) coincide con este planteamiento cuando afirma que, las actuaciones en edificios emblemáticos, aunque suponen inversión pública, son bien aceptadas y en un alto porcentaje demandadas por la población. También señala que hoy nadie discute la necesidad de rehabilitar bienes patrimoniales, si se encuentran en centros históricos, donde va a ser inmediata la rentabilidad política de una inversión realizada en un bien que se relaciona con la memoria colectiva. En este contexto, Fernández-Baca (1999) destaca que, tanto la participación de diferentes agentes en la preservación del patrimonio como el sentido de afirmación colectiva que genera, son las dos claves que potencian al patrimonio como recurso extraordinario para la cohesión social.

Para Fernández-Alba (1995: 37), el concepto de monumento y los procesos de conservación, intervención y transformación del patrimonio, atiende a diferentes valoraciones dentro de cada contexto histórico-cultural. Para este autor, la intervención en el patrimonio arquitectónico, su puesta en valor y uso, constituye hoy un campo del proyecto arquitectónico que reclama el desarrollo de un pensamiento analítico-conceptual que va más allá de la búsqueda formal, como principio artístico y valor de contemporaneidad, se trata más bien de una reflexión que se realiza desde su materialidad construida y sus acontecimientos biográficos, interrelacionados con los nuevos valores de uso, función, tecnologías y valores estéticos. El proyecto arquitectónico que aborda la intervención en un conjunto patrimonial para Fernández-Alba (1995: 45), debe considerar tres aspectos básicos: aceptar que el edificio, monumento o espacio público, tiene una microhistoria o perfil biográfico, que debe considerarse en todo el proceso de intervención; que existe una axiología del monumento o conjunto, es decir, posee un valor socio-cultural definido $\mathrm{y}$, que esta insertado en un territorio, en un paisaje urbano, que tiene características propias $y$ unos limites.

De acuerdo a Posani (1999), el patrimonio cultural debe ser preservado por lo que transmite como memoria social, por su aporte a la formación de identidad, por su valor histórico, por su capacidad de recreación y disfrute estético y por su potencial para formar parte de un plan económico. Por otro lado, Castells (1998) plantea que, junto con la revolución tecnológica, la transformación del capitalismo y la desaparición del estatismo, a finales del siglo XX experimentamos una marejada de vigorosas expresiones de identidad colectiva que desafían la globalización y el cosmopolitismo, en nombre de la singularidad cultural y del control de la gente de sus vidas y entornos. En América Latina y Venezuela la pérdida del patrimonio se debe a varios factores, entre los que destacan dos como los más importantes: la falta de interés y la ausencia de una conciencia conservacionista. 


\subsection{Rehabilitación urbana de centros históricos}

El centro de una ciudad se define comúnmente como el área conformada, geográfica y políticamente, por la ciudad antigua y es, por lo tanto, el espacio urbano con más fuerte significación. Para Precedo (1996: 243), todo centro histórico constituye un subsistema singular dentro del espacio urbano, el que mejor conserva la memoria colectiva del pasado, que debe ser transmitida a las generaciones futuras $y$, constituye un espacio singular e irrepetible dentro del tejido urbano. En este contexto, la memoria se presenta como cadencia del espacio que busca equilibrar la erosión producida en la ciudad por la colonización mercantil. Ahora, ¿Cómo encontrar la memoria en la metrópoli contemporánea? De acuerdo a Fernández-Alba (1995: 46), la respuesta en los noventa fue el reciclaje; reciclar el acontecer histórico, sus imágenes $\mathrm{y}$ formas, a través de proyectos restauradores que ponen en valor el patrimonio mediante la re-presentación de un pensamiento elaborado. Todo proyecto para Fernández-Alba (1995: 46), incluso el de la mirada restaurada, es la visión subjetivada de un cosmos. Proyectar para este autor es un peregrinaje por lo pensado, tránsito entre la ficción y la construcción, realidad de la técnica que no puede eludir la materia.

En síntesis, un centro histórico, relaciona historia y el hecho urbano, une la cultura y sus manifestaciones concretas, la cotidianidad de la vida, el trabajo y el ocio. El centro histórico de Maracaibo ocupa el área de lo que era la ciudad antes de 1900 , donde existen distintas tipo- logías edificatorias de valor histórico - patrimonial, calles y plazas, que han sobrevivido a las presiones del desarrollo contemporáneo, aunque actualmente están muy deterioradas. Las cualidades morfotipológicas y características funcionales de los centros históricos, dan carácter e identidad a estas áreas urbanas.

Un elemento importante y vital de los centros históricos es su gente, su población, la manifestación diaria de sus costumbres, el respeto a ellas, el uso de esa cotidianeidad que es el verdadero vehículo de vida de esos centros. En este sentido, la rehabilitación viabiliza la revitalización general del área, el rescate de los sentimientos, valores e identidad de la comunidad que habita esa zona de la ciudad. Lo más importante es preservar la calidad de vida y ambiental, que es en definitiva, el carácter que los grupos sociales otorgan al espacio público y/o a los edificios, por eso desde hace algunos años han surgido tendencias denominadas difusión social, que conectan la actividad de rescate patrimonial con los usuarios y requieren aplicar el concepto de sostenibilidad para el patrimonio, que no es otra cosa que no atentar contra su autenticidad.

\subsection{Gestión e Intervención Estratégica en las ciudades}

La fábrica física y el tejido socioeconómico de nuestras ciudades tienen dificultades para adaptarse a los cambios geopolíticos, económicos-sociales, tecnológicos y administrativos. Ante esta situación cabe preguntarse ¿son los actuales instrumentos de planificación y gestión urbana válidos para responder a los 
cambios del entorno? Para viabilizar las operaciones reestructuradoras del tejido físico, económico y social de las ciudades se necesita, según Fernández (1997), un proyecto estratégico que cumpla por lo menos con dos objetivos: articular las acciones sectoriales en un programa global y estimular al conjunto de la sociedad para lograr el horizonte o visión definida. En este sentido y, según Fernández Gúell (1997: 54), la planificación estratégica de ciudades es una forma sistemática de manejar el cambio y de crear el mejor futuro para la ciudad o, un proceso creativo que sienta las bases de una actuación integrada a largo plazo, a partir de un sistema continuo de toma de decisión que comporta riesgos, identifica cursos de acción específicos, formula indicadores de seguimiento para evaluar los resultadose involucrar a los agentes locales durante todo el proceso.

\subsection{Políticas Públicas y Políticas Urbanas}

Según Brugué y Gomà (1998:187), las políticas públicas aparecen como espacios centrales de mediación y resolu ción de conflictos entre colectivos con preferencias no coincidentes. En ese contexto, los problemas urbanos se encuadran también en un amplio proceso de mediación política y las políticas públicas se convierten también, en espacios de regulación y gestión del conflicto urbano. Una política es una compleja y flexible interconexión de procesos en la que los problemas y sus soluciones son constantemente redefinidos o re-inventados. Las políticas deben considerarse como propuestas de regulación pública de los mút- tiples problemas y contradicciones que afrontan las sociedades actuales. Toda política pública entraña un mecanismo de asignación pública de recursos y oportunidades entre los diferentes grupos sociales con intereses y preferencias en conflicto (Brugué y Gomà, 1998).

Las políticas, en consecuencia, implican opciones de fondo enraizadas en valores, paradigmas e ideas (Subirats, 1989 y Brugué y Gomà, 1998). Para Uvalle (2001: 311y315), las políticas públicas son por un lado, pieza esencial para los procesos de gobernación ya que, como productos del sistema político influyen en las elecciones, preferencias y expectativas de los ciudadanos $y$, por otro lado, las políticas públicas son el medio para que los ciudadanos organizados tengan presencia y representación en los procesos para democratizar el gobierno. Según este autor, gobernar por políticas implica responder a intereses ciudadanos y reconocer la pluralidad y el carácter competitivo de las demandas.

De acuerdo a Brugué y Gomà (1998: 184), las políticas urbanas constituyen la dimensión espacial del Estado de bienestar y, como forma de regulación política de la división económica y social del espacio, Ias POLÍTICAS URBANAS, tienen el reto de diseñar y poner en práctica nuevos y potentes instrumentos de regulación pública de la ciudad, si no se quiere que ésta sea el resultado estricto de la lógica del mercado. El ámbito sustantivo de la política urbana, como dimensión espacial del Estado del bienestar o como forma de regulación pública de la división económica y social del espacio, incluye los siguientes sectores de actuación pública: la localización y reestructu- 
ración económica del territorio, la regulación de usos por medio del planeamiento, la vivienda en todos sus aspectos, el transporte colectivo y los equipamientos e infraestructura conectados a la oferta de servicios públicos (Brugué y Gomà, 1998).

\section{Realizando lo imaginado: La construcción de un pacto por la ciudad}

Realizando lo imaginado alude al logro del objetivo planteado en la investigación, que incluyó la construcción de un pacto para rehabilitar el Malecón de Maracaibo. El enfoque y el método utilizado por el grupo de investigación, así como los criterios para la selección de los actores que intervienen en el mismo se describen a continuación.

\subsection{Enfoque y Método: la formación de una política bottom up}

La formación de la política urbana bottom up o construcción de un pacto por la ciudad es el resultado de una intervención urbana estratégica y participativa, promovida desde la INVESTIGACION UNIVERSITARIA, que integra la gestión urbana con la intervención social, para generar nuevos métodos y prácticas urbanas que viabilizan el proceso de construcción de la ciudad. El objeto de este proceso es construir -en forma concertada y colectiva- la viabilidad política, institucional, social-cultural y financiera, al proyecto de rehabilitación de El Malecón. En este contexto, el proceso de viabilización tiene dos objetivos básicos: compatibilizar las expectativas de los diferentes actores sociales con las propuestas que se generen y, establecer nuevos patrones de conducta y, una nueva cultura urbana, para inducir a la toma de decisión en forma compartida y colectiva (Ferrer y Avila, 1997).

En este sentido, el MODELO DE GESTIÓN implantado (Cuadro 1), asume la intervención urbana como proceso de negociación y gestión coordinada y continua entre diferentes actores, públicos, privados y asociativos, para seleccionar por consenso vías para solucionar los problemas percibidos en el uso cotidiano de las ciudades (Ferrer, 1995), en este caso la puesta en valor de El Malecón de Maracaibo. La garantía de éxito del proceso de intervención propuesto se centró en movilizar la confianza activa (Fukuyama, 1996), a través de la participación democrática y sinérgica de los actores, para que se hicieran corresponsables de la rehabilitación de El Malecón.

EI MODELO DE GESTIÓN desarrollado se apoyó inicialmente en el método Ferrer y Avila (1997), que se complementó para iniciar el proceso de viabilización ex - antes y durante la construcción de la política urbana bottom up o pacto por la ciudad. El nuevo Modelo de Gestión se estructuró en tres Fases: la Fase 1: Planificación de la Gestión, definición del curso de acción o estrategia operativa, para promover la actuación coordinada interinstitucional; Fase 2: Construcción de Consensos o, visión compartida, que incluyó el diseño del convenio para la rehabilitación de El Malecón como estrategia colectiva y, la Fase 3: Ejecución del Convenio de Gestión o pacto por la ciudad, que se desarrolló en dos etapas (Cuadro 2), Etapa 1: Compromiso de 


\section{Cuadro 1 \\ Modelo de gestión para la Rehabilitación del Malecón}

Fases

Fase 1

PLANIFICACIÓN

DE LA GESTIÓN

(Formulación consensuada de la política y estrategia operativa)

\section{Fase 2}

CONSTRUCCIÓN

DEL CONSENSO

(visión compartida)

\section{Objetivos}

Productos

Definir la estrategia operativa para promover la actuación coordinada interinstitucional en EI MALECÓN

\section{Estrategia implantada \\ Logrado acuerdo}

Elaborar concertadamente: Convenio de Gestión Estratégica

a. El Convenio de Gestión Bases del Concurso

Estratégica b. Las Bases del

Concurso Internacional de Ideas

\section{Fase 3}

EJECUCIÓN CONVENIO GESTIÓN

(pacto por la ciudad)
Convenio firmado

Firmar el CGE*

Ejecutar el CGE
Concurso Internacional realizado

Propuesta de Ideas seleccionada Contratación del proyecto

Fuente: Elaboración propia.

* Convenio de Gestión Estratégica.

\section{Cuadro 2}

\section{Etapas y objetivos de la fase 3 del modelo de gestión}

\begin{tabular}{l|c|c}
\hline \multicolumn{1}{c|}{ Fase 3 } & Etapas & \multicolumn{1}{c}{ Objetivos } \\
\hline $\begin{array}{l}\text { EJECUCIÓN CONVENIO } \\
\text { GESTIÓN } \\
\text { (pacto por la ciudad) }\end{array}$ & $\begin{array}{c}\text { Etapa 1: Compromiso de } \\
\text { participación } \\
\text { Etapa 2: Corresponsabilidad } \\
\text { en la Gestión }\end{array}$ & $\begin{array}{l}\text { Firmar el Convenio de Gestión } \\
\text { Ejecutar el Convenio de Gestión }\end{array}$ \\
\hline
\end{tabular}

Fuente: Elaboración propia.

participación, logrado con la firma del Convenio de Gestión Estratégica, CGE, para la Rehabilitación de El Malecón y su Entorno Inmediato y, la Etapa 2: Corresponsabilidad en la Gestión, que es el proceso implantado para el logro de los tres objetivos del Convenio.

\subsection{Caracterización y selección de actores}

En relación con los agentes intervinientes, coincidimos con Fernández
(1997), cuando dice que, para poner en marcha un proceso de participación es necesario comprender los intereses de los actores e involucrarlos en el proceso de planificación. En definitiva, una intervención estratégica urbana debe ser capaz de dar cabida a los diferentes agentes locales y, supralocales, que intervienen en los procesos de desarrollo urbano $y$, conciliar sus intereses, a partir de la formulación de una estrategia concertada de futuro para la comunidad. En este sen- 
tido, Fernández-Baca (1999), refuerza este planteamiento cuando señala que, la tendencia en el discurso patrimonial actual es la necesaria intervención de multitud de actores para el correcto tratamiento de los bienes culturales.

En este contexto y con el objetivo de seleccionar e invitar a participar a los actores en el CONVENIO DE GESTION ESTRATEGICA se consideraron tres aspectos (Ferrer, 1995): los objetivos de la investigación, las competencias asignadas a las instituciones en la legislación vigente y, la actividad o función que realizan los actores o podrían realizar en el ámbito de la ejecución del proyecto, es decir: quién decide, planifica, financia, ejecuta, mantiene y/o controla. También se consideraron los criterios siguientes: competencia y/o interés en el proyecto; responsabilidades políticas; responsabilidades gremiales; representación de sectores económicos, sociales y culturales de la ciudad; representantes de Asociaciones de Vecinos, ONG's; nivel técnico y asesores de las diferentes instituciones.

Los actores que participan en el proyecto son, a nivel regional: La Universidad del Zulia a través del grupo de investigación de la FAD y del Centro de Estudios de la Empresa (CEE), de la Facultad de Ciencias Económicas y Sociales (FCES), el Centro Rafael Urdaneta (CRU), encargado de la promoción y gerencia del Area Central de Maracaibo, entre otras competencias; la Secretaria de Cultura del Estado Zulia encargada de la declaratoria de EI Malecón como patrimonio regional; El Puerto de Maracaibo insti- tución que cedió en comodato al $\mathrm{CRU}$ el terreno de El Malecón; las Cámaras de la Construcción e Inmobiliaria interesadas en la promoción y revitalización del área central de Maracaibo y, La Unión de Comerciantes e Industriales del Zulia, institución a la que pertenecen muchos de los comerciantes del área central. A nivel local participan: la Alcaldía de Maracaibo interesada y con competencia en el Area Central de Maracaibo (ACM), la Cámara de Comercio interesada en impulsar y revitalizar la actividad comercial; El Centro de Arte de Maracaibo Lía Bermúdez (CAMLB), cuya sede de valor histórico patrimonial (antiguo mercado de la ciudad), forma parte del espacio de el Malecón y es un actor involucrado e interesado en su puesta en valor y, la Asociación Civil, Centro de Información Patrimonial el Tabacal, ubicada en Santa Lucia, zona residencial patrimonial adyacente a el Malecón, interesada en promover un proceso similar en este sector de la ciudad. A nivel nacional: el Instituto de Patrimonio Cultural, IPC, institución rectora encargada de la salvaguarda del patrimonio cultural y natural del país (Ver Cuadro 3).

La selección de los actores también consideró su pertinencia para la construcción de la viabilidad política, institucional, social-cultural y económico-financiera, al proceso de rehabilitación de el Malecón. En este sentido, en el Cuadro 4 se explicita la relación actores-viabilidad. Los actores se ubican de acuerdo a su competencia, poder y a la viabilidad que se espera lograr con su participación en el proyecto. 


\section{Cuadro 3 \\ Modelo de gestión, fases y actores del Convenio de Gestión Estratégica (CGE)}

Modelo de gestión: Fases

Fase 1

\section{Actores}

Grupo de Investigación

LUZ

Comisión Ejecutiva CGE:

Alcaldía de Maracaibo, CRU S.A, Cámaras: Construcción, In-

Fase 2 mobiliaria y Comercio, CAMLB, Secretaría de Cultura, Centro de Información Patrimonial El Tabacal, IPC, LUZ,

Puerto de Maracaibo, UCEZ

Consejo Directivo CGE

Comisión Ejecutiva CGE

Comité de Administración

Fuente: Elaboración propia.

\section{Cuadro 4}

Relación viabilidad-actores Convenio de Gestión Estratégica (CGE)

\begin{tabular}{ccccc}
\hline Viabilidad & Política & Institucional & $\begin{array}{c}\text { Social-Cultural } \\
\text { (Sociedad } \\
\text { Organizada) }\end{array}$ & $\begin{array}{c}\text { Econ-Financiero } \\
\text { (Promotores) }\end{array}$ \\
\hline A & Gobernador & CRU & CRU \\
$\mathbf{C}$ & Alcalde & OMPU, IMTCUMA & Asociaciones & Puerto \\
$\mathbf{T}$ & Concejales & C. Comercio & CAMLB & Proyectistas \\
$\mathbf{0}$ & Líderes Políticos & IPC & Gremios & \\
$\mathbf{R}$ & Grupo & C. Inmobiliaria & ONG'S & Asamblea Nacional \\
$\mathbf{E}$ & Parlamentario & C. Construccion & & PNUD \\
$\mathbf{S}$ & Junta Parroquial & Secretaria de Cultura & & Constructores \\
\hline
\end{tabular}

Fuente: Elaboración propia, a partir de Ferrer (1995) y Ferrer y Avila (1997).

OMPU Oficina Municipal de Planeamiento Urbano.

IMTCUMA Instituto Municipal de Transporte Urbano de Maracaibo

CAMLB Centro de Arte de Maracaibo, Lía Bermúdez 


\subsection{Conformación de la Estructura Organizativa del Convenio}

Una de las causas principales de las dificultades que confrontan las Instituciones para viabilizar la concreción de sus planes y proyectos, es la ausencia de una estructura organizativa - no burocrática - capaz de asumir responsablemente en el proceso de gestión y, de canales, que permitan optimizar la participación sinérgica de los actores. En este sentido la estructura organizativa del Convenio incluye tres niveles de actuación: un Consejo Directivo, conformado por los presidentes de las instituciones signatarias del Convenio, una Comisión Ejecutiva, conformada por técnicos de las instituciones y el grupo de investigación de la FAD $y$, un Comité de Administración, conformado por siete representantes de las instituciones y cinco suplentes, especialistas en esta materia. El proceso dialogante implantado durante las dos etapas de esta investigación extensiva ha implicado y permitido el surgimiento de una nueva cultura ciudadana, donde se comparten responsabilidades, liderazgos y compromisos y, desarrollar nuevos enfoques y metodologías de gestión participativa. En este proceso, la participación del grupo de investigación aplicada de la FAD ha sido clave como promotores de la idea inicial y en la concreción del proyecto.

\section{Conclusiones y reflexiones finales}

En este punto se señalan las conclusiones más importantes en relación con dos aspectos: el proceso implantado y, con la investigación aplicada universitaria, indicando los logros obtenidos hasta la fecha y se incluyen unas reflexiones finales.

En cuanto a los aspectos del proceso que merecen destacarse:

- El modelo de gestión diseñado e implantado, concilió la gestión pública con la iniciativa privada y asociativa, generando un aprendizaje colectivo desde la experiencia donde, doce actores, tradicionalmente en conflicto y compitiendo para impulsar proyectos individuales, comparten liderazgo y, objetivos a través del Convenio de Gestión Estratégica para la Revitalización de el Malecón de Maracaibo y su entorno inmediato, con el fin de rescatar colectivamente el Malecón

- El proceso dialogante implantado durante las dos etapas de esta investigación ha implicado y permitido el surgimiento de una nueva experiencia institucional y ciudadana, donde se comparten responsabilidades, liderazgos y compromisos.

- Durante la investigación, se han desarrollado y aplicado nuevos enfoques y metodologías de gestión participativa, que han viabilizado la formación de una política urbana bottom up (de abajo arriba).

- En el proceso, la participación del grupo de investigación de la FADLUZ, ha sido clave como promotores de la idea inicial y en la concreción del proyecto.

En cuanto a la investigación aplicada universitaria se puede concluir:

- El potencial estratégico de la investigación aplicada o extensiva para generar impacto social. 
- La emergencia de una nueva praxis universitaria, postburocratica, plural y posfuncionalista, que considera la investigación, docencia y extensión como actividades inescindibles.

- La necesidad de conformar y trabajar en redes interinstitucionales que potencien la vinculación público-privado y asociativo.

- La necesidad de involucrar a diferentes actores sociales en el proceso de formación de la política urbana, como auténtica clave para desarrollar comunidades y ciudades sostenibles.

Como logros importantes se pueden señalar los siguientes: el desarrollo e implantación de un modelo o mecanismo de gestión que permitió la concreción de los dos primeros objetivos del Convenio $\mathrm{y}$, el avance en la declaratoria de El Malecón como bien patrimonial de la Región Zuliana, que está en proceso de aprobación por las instancias correspondientes. Por otro lado, debe destacarse el esfuerzo realizado en la formación del equipo del Convenio y, en el proceso comunicacional desarrollado para informar a la comunidad y viabilizar el proyecto, que se ha concretado mediante la realización de seminarios, exposiciones, charlas y conferencias así como, a través de la incorporación de expertos, tanto nacionales como extranjeros (de España y Francia), como asesores del proyecto y/o, mediante su participación como jurados en el Concurso Internacional de Ideas. La participación del asesor de Francia se logró a través del Convenio suscrito entre la Comunidad de Burdeos y la Alcaldía de Maracaibo.

Esta experiencia ha hecho patente la necesidad de formar nuevos colectivos profesionales en diferentes áreas pero especialmente para el tratamiento y gestión del hecho urbano y, específicamente del patrimonio urbano (natural y cultural), así como de interventores sociales. La formación de gestores urbanos y del patrimonio, entre otros, requiere la continua y permanente acción formativa universitaria adaptada a las nuevas realidades que envuelven nuestras ciudades, tarea que puede acometerse desde la investigación, la formación continua y desde los programas de postgrado, entre otros.

Entre los retos que quedan pendientes destacan tres: continuar el proceso hasta concretar la rehabilitación de EI Malecón y su entorno inmediato; promover soluciones imaginativas que fomenten la utilización continuada de El Malecón y, así evitar que con el tiempo sea necesario rehabilitar lo rehabilitado $\mathrm{y}$, conseguir incentivos sociales y económicos a las inversiones en este contenedor urbano-patrimonial (edificaciones y espacio público) para dotarlo de vitalidad, con la participación activa de la pluralidad de agentes sociales interesados e involucrados en la recuperación del área central de Maracaibo.

\section{Bibliografía Citada}

Berlinches Acin, Amparo (1995), La rehabilitación del patrimonio en tres Centros Históricos de Madrid. En Intervención Estratégica en la Ciudad. En ciudades Iberoamericanas y España. Comunidad de Madrid. Conserjería de Política Territorial. Colección Documentos Técnicos. Madrid.

Brugue, Quim y GOMA, Ricard (1998), Gobiernos locales y políticas públicas. Bienestar social, promoción económica y territorio. Ariel Ciencia Política. Barcelona/España. 
Castells, Manuel (1998), La era de la información. Vol.III. Fin de Milenio.Alianza Editorial.

Crozier, Michel (1996), La crisis de la inteligencia, ensayo sobre la incapacidad de las elites para reformarse. Publicación del Ministerio de Administración Publica, MAP. Madrid.

Cruz, Edgard (2001). Civilización o Barbarie. En, El Universal, ZONA, p2, 30 de Septiembre.

Fernández-Alba, Antonio (1995), Patrimonio y Proyecto Arquitectónico. En, Revista Astrágalo. No. 3 Septiembre. HISTORIA Y PROYECTO. Instituto Español de Arquitectura. Universidades de Alcalá y Valladolid. Editorial Celeste. España.

Fernández - Baca, Román (1999), Patrimonio Histórico, cohesión social e innovación. En, Boletín del Instituto Andaluz del Patrimonio Histórico. Año VII, Número 27. Sevilla. España.

Fernández Guell, José Miguel (1997), Planificación Estratégica de Ciudades. Editorial GG. SA.

Fernández, Roberto (1995), Monumento y Proyecto Moderno. En, Revista Astrágalo. No. 3 Septiembre. HISTORIA Y PROYECTO. Instituto Español de Arquitectura. Universidades de Alcalá y Valladolid. Editorial Celeste. España.

Ferrer, Mercedes (1995), Modelo de Gestión: La praxis de la construcción del Plan Municipal y la Ciudad. Trabajo de Ascenso profesora Titular, FALUZ. Maracaibo. Venezuela.

Ferrer, Mercedes; AVILA, Nancy et alt (1997), Plan de Gestión de Tamare, Fase II, Etapa 1 "Relaciones con la Comunidad". Convenio LUZ-LAGOVEN-ALCALDÍAS DE LA COLM-ACIVEGESUR. Facultad de Arquitectura, LUZ. Maracaibo. Venezuela.

Ferrer, Mercedes (1998), Investigación en Extensión. Memorias, V Seminario de
Investigación. LUZ. Vicerrectorado Académico. CONDES. Maracaibo.12 al 15 de Mayo. Venezuela.

Ferrer, Mercedes (1999), Extensión en un contexto relacional. Memorias, 1er. Seminario de Extensión FADLUZ. DEAR. EDILUZ. Maracaibo. Venezuela.

Fukuyama, Francis (1996), CONFIANZA (Trust). Las virtudes sociales y la capacidad para generar prosperidad. Editorial Atlantida. Buenos Aires.

Gates, Bill (1999), Los negocios en la era digital. Plaza \& Janes. SA. E.U.A.

Giddens, Anthony (1996), Más allá de la izquierda y la derecha. El futuro de las políticas radicales. Ediciones Cátedra. SA. Madrid.

Licha, Isabel (1992), Los peligros para las universidades latinoamericanas de fin de siglo. En Cuadernos del CENDES No. 21. Segunda Epoca. Septiembre Diciembre. Caracas.

Posani, Juán Pedro (1999), Lineamientos de política de conservación. Instituto de Patrimonio Cultural, Ministerio de la Secretaría de la Presidencia. Caracas/Venezuela.

Precedo Ledo, Andrés (1996), Ciudad y desarrollo Urbano. Editorial Síntesis. Madrid. España.

Subirats, Joan y Gomá, Ricard (2000), "Cambios en las políticas de bienestar e innovación en la Unión Europea". En Revista CLAD, Reforma y Democracia, No. 17. Caracas.

Subirats, Joan (1989), Análisis de Políticas Públicas y eficacia de la administración. Instituto Nacional de Administración Pública. Madrid.

Uvalle, Ricardo (2001), "Importancia de las políticas públicas en el desarrollo complejo de la sociedad contemporánea". En Revista Venezolana de Gerencia, Año 6 - No. 14. Maracaibo, Venezuela. 